\title{
Study of System Design Parameters for Space-Based Traffic Monitoring
}

\author{
Martina Gabele*, S. Baumgartner*, G. Krieger*, K.-H. Bethke* \\ *Microwaves and Radar Institute, German Aerospace Centre (DLR) \\ Münchner Straße, 20, 82234 Weßling, GERMANY \\ email: Martina.Gabele@dlr.de
}

\begin{abstract}
This paper addresses the ground moving target indication (GMTI) performance and the impact of system design parameters on space-based multi-channel synthetic aperture radar (MSAR) systems as they suffer from the high speed of the radar platform leading to a wide spread clutter spectrum. Opposite to classical GMTI systems near future space-based SAR systems offer only few simultaneous channels and relatively low pulse repetition frequency $(P R F)$. The impact of PRF, antenna size, antenna separations and number of channels on detection performance is studied. The analysis is based on a post-Doppler approach of the optimal processor.
\end{abstract}

\section{Introduction}

Traditionally GMTI has been applied to military detection and observation of few targets. A new field of GMTI is traffic monitoring. Traffic monitoring requires snapshots of huge area traffic scenes with high repeat cycle from which traffic density and travelling velocity can be determined. The Traffic Monitoring with Air- and Space-based Radar (TRAMRAD) project aims to find system concepts which are specialized to traffic monitoring [1].

The contribution of this paper is to investigate the impact of $P R F$, receiver antenna size, antenna separations and number of channels on detection performance. Starting from a single satellite system it is shown that future GMTI satellites need to exhibit large antenna configurations. Hence, the receiver array of the single satellite system is extended in alongtrack direction by use of a boom. Effective receiver array extensions in the same order or larger can be achieved with TanDEM-X [2], an actual future multi-channel SAR system consisting of two TerraSAR-X [3] satellites flying in close formation.

\section{Signal and Clutter Model}

For the analysis we use a post-Doppler approach of the optimal processor [4]. Hence, for stationary Gaussian clutter the clutter contributions can be assumed uncorrelated for each

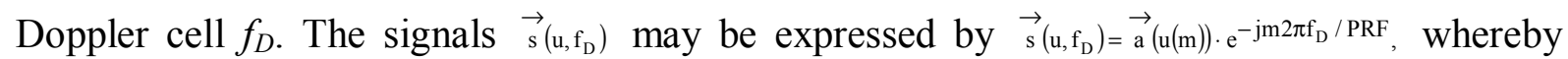
$\mathrm{e}^{-\mathrm{jm} 2 \pi \mathrm{f}_{\mathrm{D}} / \mathrm{PRF}}$ describes the temporal sampling given by the index $m$

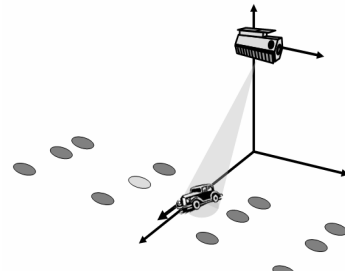

Figure 1 Moving target and corresponding (bright ellipse) as well as ambiguous clutter patches (dark ellipses). and $\vec{a}(u(m))$ contains the two-way antenna pattern weighting and the phase shifts between the receivers due to the distance of the effective phase centres to the origin. The target signal Doppler frequency $f_{D}$ depends on the velocity $v_{y}$ and the direction $u_{t}(m)$ of the target. However there is also a corresponding clutter cell on ground, which contains the same Doppler frequency $f_{D}$ as the moving target but comes from a different direction $u_{c c}(m)$. The corresponding clutter patch is sketched in Figure 1 as a bright ellipse on ground. There are even more ambiguous clutter patches on the ground containing the same Doppler frequency $f_{D}$ but 
arriving from directions $u_{a c, i}(m)$ due to temporal subsampling and range ambiguities. Their position and their number are dependent on the $P R F$ : for low $P R F$ the ambiguous clutter patches are more dense in azimuth, for high $P R F$ they are more dense in range. The ambiguous clutter patches are shown as dark ellipses on the ground. The clutter contributions to one target Doppler frequency $f_{D}$ can be modelled by the clutter contributions from the corresponding and the ambiguous clutter cells $\vec{s}\left(u+f_{1}, f_{D}\right)$. Hence, the clutter plus noise covariance matrix $C_{W}$ can be determined as follows: $\mathrm{c}_{\mathrm{w}}\left(\mathrm{f}_{\mathrm{D}}\right)=\mathrm{\sigma}_{\mathrm{N}}^{2} \cdot \mathrm{I}+\sum_{\mathrm{i}} \mathrm{P}_{\mathrm{c}} \cdot \vec{s}\left({\overrightarrow{\mathrm{u}_{\mathrm{i}}}}_{\mathrm{i}}, \mathrm{f}_{\mathrm{D}}\right) \cdot\left(\overrightarrow{\mathrm{s}}^{*}\left(\mathrm{u}_{\mathrm{u}_{\mathrm{i}}}, \mathrm{f}_{\mathrm{D}}\right)\right)^{\mathrm{T}}$, where $I$ is the identity matrix, $\sigma_{N}^{2}$ is the noise power, $P_{c}$ the clutter power and the sum sign describes the summation over the matrices formed by the dyadic products of the sample vectors from each clutter cell. The signal to clutter and noise ratio $(S C N R)$ at the output of the optimal filter can be determined by:

$$
\operatorname{SCNR}\left(u_{t}, f_{D}\right)=P_{S} \cdot \vec{s}\left(u_{t}, f_{D}\right) \cdot C_{W}^{-1}\left(f_{D}\right) \cdot \vec{s}^{*}\left(u_{t}, f_{D}\right) \cdot
$$

Since the resulting detection performance is a function of the $S C N R$ we use it in the following for detection performance analysis. For analysis we assume a normalized $S N R=0 d B$ and $C N R=20 \mathrm{~dB}$ per temporal sample independently of the receiver aperture size.

\section{System Pre-Considerations}

\section{System Requirements:}

A traffic monitoring system should at least be capable of detecting vehicles moving with ground velocities between $30 \mathrm{~km} / \mathrm{h}$ and $200 \mathrm{~km} / \mathrm{h}$. Since the radar cross section (RCS) of vehicles strongly varies with aspect angle [1], a traffic monitoring system should be able to detect targets with an RCS of down to $-10 \mathrm{dBm}^{2}$. Fulfilling these constraints the system should cover a search area as large as possible.

\section{Basic System Parameters:}

We start from a single satellite system concept in X-band similar to TerraSAR-X [3]: The slant range at an incidence angle of $\theta=45^{\circ}$ we assume to $R_{S}=700 \mathrm{~km}$ and the platform velocity to $v_{P}=7300 \mathrm{~m} / \mathrm{s}$. The average transmit power is $P_{T x}=400 \mathrm{~W}$, the system noise temperature being $T_{S}=300 \mathrm{~K}$, and the system noise figure $F=5 \mathrm{~dB}$. To unambiguously cover all motion directions on ground a $P R F=6 \mathrm{kHz}$ is required. Hence, in order to avoid range ambiguities within the null-to-null antenna mainbeam the antenna height shall be $L_{R}=$ $0.9 \mathrm{~m}$. Applying the radar equation this results in an antenna length in azimuth of minimum $L_{A z}=20 \mathrm{~m}$ on transmit and receive and the full $3 \mathrm{~dB}$-beamwidth integration time in stripmap mode in order to achieve a signal to noise ratio after coherent integration of $S N R=20 \mathrm{~dB}$.

\section{Comparison of Systems}

\subsection{Single Satellite System}

In Figure 2 the impact of partitioning the whole receiver aperture of $L_{A z}=20 \mathrm{~m}$ into equally large subapertures is studied. The $S C N R$ was first determined for each Doppler bin and then integrated along all Doppler bins during $3 d B$ integration time. On the abscissa the line-ofsight (LOS) across-track velocity $v_{y}$ is shown. As can be seen, the system performs almost equally well for two and more subapertures with respect to detectability. The $-3 d B$ minimum detectable velocity $(M D V)$ in LOS which is dominated by the maximum array extension is 


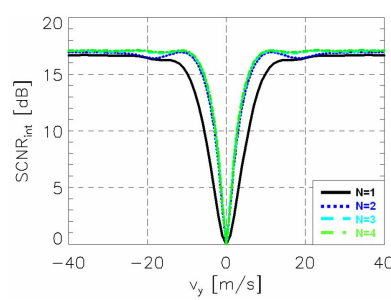

Figure 2 Impact of partitioning receiver antenna into $N$ equal subapertures on $S C N R_{\text {int }}$ (SCNR integrated along the $3 d B$ beamwidth).
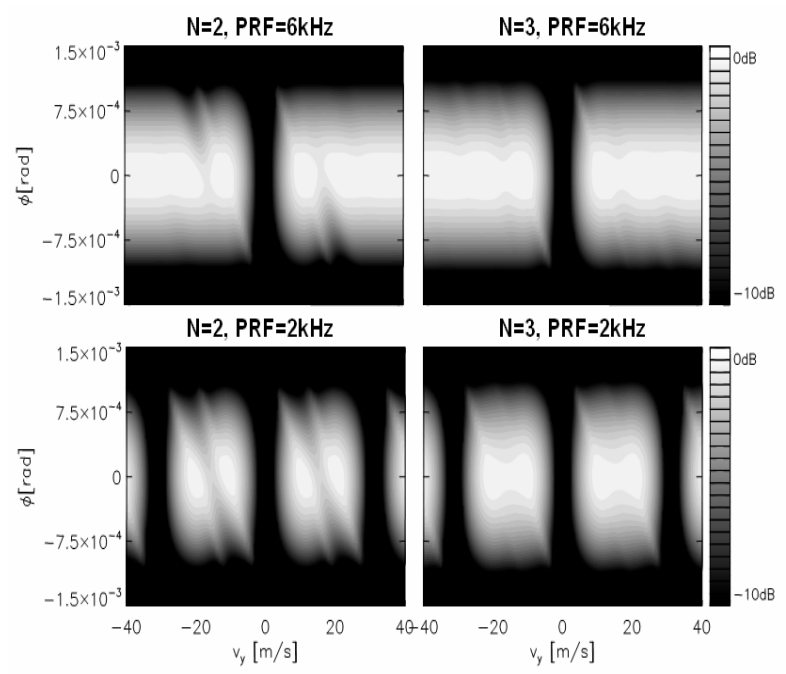

$\mathrm{N}=3, \mathrm{PRF}=2 \mathrm{kHz}$

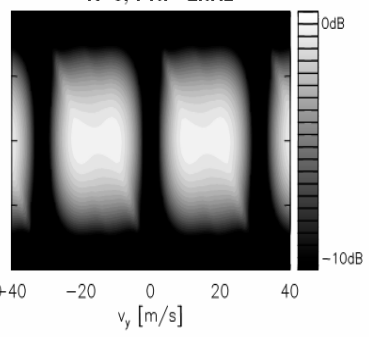

Figure 4 Comparison of degradations of two / three channel system due to ambiguous clutter patches.
$M D V=5 \mathrm{~m} / \mathrm{s}$. This transforms to a minimum detectable ground velocity with an acrosstrack component of $v_{y, \text { ground }}=25 \mathrm{~km} / \mathrm{h}$. Hence, the system requirements are fulfilled only for streets which run almost orthogonal to the flight path.

Another drawback is the swath width on ground which is limited to $S W_{\max }=35 \mathrm{~km}$. Increasing the swath width by lowering the $P R F$ up to $P R F=3 \mathrm{kHz}$ works well besides the fact that additional information about target velocities is required since the velocities are ambiguous. Further lowering causes severe drawbacks in many respects: blind velocities occur at $\mathrm{vblind}=\frac{\lambda}{2} \mathrm{PRF}$, multiple fold-backs of target velocities, and increasing impact of ambiguous clutter patches since the grid width of clutter ambiguities in azimuth is proportional to $P R F$ (see Figure 1); last but not least swath widening reduces $S N R$ by reduction of antenna gain. In Figure 3 the impact of lowering the $P R F$ is shown: First, a $P R F=2 \mathrm{kHz}$ severely reduces the detectable target velocity range by placing a blind velocity $v_{\text {blind }}=31 \mathrm{~m} / \mathrm{s}$. In the detectable target velocity range the two-channel system shows further degradations in case that $P R F$ $=2 \mathrm{kHz}$ due to the fact that more than one clutter patch may be placed within the null-

to-null mainbeam width of the antenna while the platform is passing the scene. A comparison of the contamination due to ambiguous clutter patches dependent on the number of subapertures $N$ during the $3 d B$ integration time is shown in Figure 4.

\subsection{Single Satellite with Boom}

For reduction of the $M D V$ an extension of the receiver array, or more precisely the effective phase centres is necessary. On a single satellite this can be accomplished by use of a boom and aperture switching on transmit: If aperture switching on transmit is introduced such that transmission is switched between the leading and the trailing antenna (reception with all subapertures), an unequally spaced effective phase centre configuration with increased separations but halved effective PRF results. As long as the Nyquist condition for azimuth sampling is fulfilled for the effective PRF, restoring the situation of simultaneous sampling is possible. The same effective phase centre configuration can also be achieved without halving the effective $P R F$ by parallel transmission of orthogonal waveforms with the leading and trailing antenna. In Figure 5 the $S C N R_{\text {int }}$ for the equivalent simultaneous sampling is shown for the dual channel single satellite system described above extended by another subaperture of length $L=10 \mathrm{~m}$ separated in along-track direction by various boom lengths such that the maximum receiver array extension is $L_{A z}=60 \mathrm{~m}, 50 \mathrm{~m}$, and $40 \mathrm{~m}$. The minimum detectable across-track velocity component in LOS direction reduces to $v_{y}=1-2 \mathrm{~m}$ and in case that the 


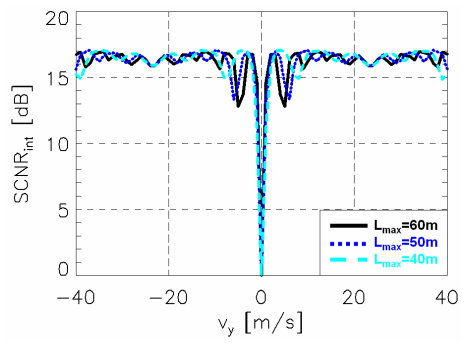

Figure $5 S^{S C N R_{\text {int }}}$ for $P R F_{\text {eff }}=3 \mathrm{kHz}$ and increase of effective phase centre separation using a boom and aperture switching on transmit (equivalent simultaneous sampling).

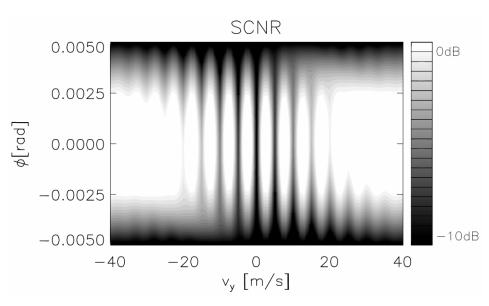

Figure $6 S C N R_{\text {int }}$ plane of TanDEM-X with both satellites in dual receive antenna mode (maximum receiver array extension: $50 \mathrm{~m}, P R F=6$ $k H z)$. receiver array extension is accomplished by transmission of orthogonal waveforms a real $P R F=3 \mathrm{kHz}$ is sufficient enabling a swath width on ground of $70 \mathrm{~km}$.

\subsection{Dual Satellite System TanDEM-X}

Another way to increase the receiver array extension is to distribute the receiver array on several satellites as will be possible with TanDEM-X [2], a near-future SAR system consisting of two TerraSAR-X satellites [3] flying in close formation. Analyses showed that the system performs best in case that the DPCA condition is fulfilled for each satellite enabling a system of four parallel receiver channels with a small baseline on each satellite and a large baseline in between the satellites. In Figure 6 both satellites are operated in dual channel mode with $P R F=6 \mathrm{kHz}$ and are separated in along-track direction such that a maximum receiver array extension of $L_{A Z}=$ $50 \mathrm{~m}$ results. The $S C N R$ plane strongly varies with target velocity due to gratinglobes introduced by the large satellite separation and lead to performance degradations down to two channel TerraSAR-X performance for certain equidistant target velocities over the whole integration time. The velocities for which performance degrades can be varied by variation of transmit frequency or satellite separation.

\section{Summary}

We studied the system requirements of a traffic monitoring system on $P R F$, receiver antenna size, antenna separations and number of channels on detection performance. The single satellite system extended with a boom and receiver array extension as well as systems where the receiver array is distributed on several satellites as e.g. with TanDEM-X seem to be promising system concepts. The analysis should also be extended to the motion parameter estimation accuracy.

\section{Reference:}

[1] D. Hounam, S. Baumgartner, K.-H. Bethke, M. Gabele, E. Kemptner, D. Klement, G. Krieger, G. Rode, K. Wägel, "An autonomous, non-cooperative, wide-area traffic Monitoring System using space-based Radar", IGARSS 2005, Seoul, Korea, 25-29.07.2005.

[2] A. Moreira, G. Krieger, I. Hajnsek, D. Hounam, M. Werner, S. Riegger, E. Settelmeyer, "TanDEM-X: A TerraSAR-X Add-On Satellite for Single-Pass SAR Interferometry", IGARSS, 2004, Anchorage, Alaska, USA, 20-24.09.2004.

[3] M. Suess, S. Riegger, W. Pitz, R. Werninghaus, "TerraSAR-X - Design and Performance", EUSAR, 2004, Ulm, Germany, 25-27.05.2004.

[4] J.H.G. Ender, D. Cerutti-Maori, W. Bürger, "Radar antenna architectures and sampling strategies for space based moving target recognition", IGARSS, 2005, Seoul, Korea, 2529.07.2005. 